MATEC Web of Conferences 33, 03013 (2015)

DOI: $10.1051 /$ matec conf/ 20153303013

(c) Owned by the authors, published by EDP Sciences, 2015

\title{
Model of the Evolution of Deformation Defects and Irreversible Strain at Thermal Cycling of Stressed TiNi Alloy Specimen
}

\author{
Aleksandr E.Volkov ${ }^{1 a}$, Fedor S.Belyaev ${ }^{1}$, Margarita E.Evard ${ }^{1}$, Natalia A.Volkova ${ }^{1}$ \\ ${ }^{1}$ Saint Petersburg State University, 198504 Saint Petersburg, Russia
}

\begin{abstract}
This microstructural model deals with simulation both of the reversible and irreversible deformation of a shape memory alloy (SMA). The martensitic transformation and the irreversible deformation due to the plastic accommodation of martensite are considered on the microscopic level. The irreversible deformation is described from the standpoint of the plastic flow theory. Isotropic hardening and kinematic hardening are taken into account and are related to the densities of scattered and oriented deformation defects. It is supposed that the phase transformation and the micro plastic deformation are caused by the generalized thermodynamic forces, which are the derivatives of the Gibbs' potential of the two-phase body. In terms of these forces conditions for the phase transformation and for the micro plastic deformation on the micro level are formulated. The macro deformation of the representative volume of the polycrystal is calculated by averaging of the micro strains related to the evolution of the martensite Bain's variants in each grain comprising this volume. The proposed model allowed simulating the evolution of the reversible and of the irreversible strains of a stressed SMA specimen under thermal cycles. The results show a good qualitative agreement with available experimental data. Specifically, it is shown that the model can describe a rather big irreversible strain in the first thermocycle and its fast decrease with the number of cycles.
\end{abstract}

\section{Introduction}

Operational integrity of actuators, sensors, vibration isolators based on the use of shape memory alloys (SMA) substantially depends on the irreversible shape change, which develops at repeated thermocycling of an SMA part subject to the action of external forces. Thus, calculation of the irreversible deformation is a challenge for any theoretical model aimed at the simulation of the SMA mechanical behavior. A suitable approach can be microstructural modeling since it is based on the account of the alloy structure and of the deformation mechanisms. Probably the first attempt to develop such a model was the work of Q. Sun and C. Lexcellent [1] who introduced special internal variables to specify the measures of the plastic deformation. Similar internal variables were used in $[2,3]$. In these works it was shown that such approach could describe an incomplete strain recovery on heating. It also naturally explained the relation between the unrecovered strain and the two-way shape memory effect. In works $[4,5]$ a further step was done. Microplastic deformation was calculated alongside with the densities of the scattered and oriented deformation defects, the isotropic and kinematic hardening being related with these densities. In the present work a more accurate account of the oriented defects is done. The density of these defects is considered to be a state variable, characterizing the elastic energy of the inter-phase stresses, rather than the irreversible deformation. The developed model is applied for simulating the variation of the irreversible deformation on thermocling under different stresses acting on heating and cooling.

\section{Model}

This microstructural model uses the approximation of the small-strain theory. The representative volume is considered to consist of grains characterized by the orientation $\omega$ of the crystallographic axes. Inside each grain coexist the austenite and martenside, which in turn is composed of domains belonging to one of the $N$ crystallographically equivalent orientations.

\subsection{Averaging of microstrains}

Reuss' hypothesis is used for calculation of the strain tensor $\varepsilon$ of the representative volume by neutralization of the strains of grains belonging to this volume:

$$
\varepsilon=\Sigma f_{i} \varepsilon^{\mathrm{gr}}\left(\omega_{\mathrm{i}}\right)
$$

where $f_{i}$ and $\varepsilon^{\mathrm{gr}}\left(\omega_{i}\right)$ are the volume fraction and the strain of a grain with the orientation of the crystallographic axes $\omega_{i}$ and the sum is taken over all grains. A grain strain $\varepsilon^{\text {gr }}$ is considered as the sum of elastic $\varepsilon^{\text {gre }}$, thermal $\varepsilon^{\text {gr T }}$, phase $\varepsilon^{\mathrm{grPh}}$ and micro plastic $\varepsilon^{\mathrm{gr} \mathrm{MP}}$ components:

$$
\varepsilon^{\mathrm{gr}}=\varepsilon^{\mathrm{gre}}+\varepsilon^{\mathrm{grT}}+\varepsilon^{\mathrm{grPh}}+\varepsilon^{\mathrm{gr} \mathrm{MP}}
$$

\footnotetext{
a Corresponding author: author@e-mail.org
} 
Also within the Reuss' hypothesis, we calculate the elastic strain of a grain by the "mixture rule":

$$
\varepsilon^{\mathrm{gre}}=\left(1-\Phi^{\mathrm{gr}}\right) \varepsilon^{\mathrm{A} \mathrm{e}}+\frac{1}{N} \sum_{n=1}^{N} \Phi_{n} \varepsilon^{\mathrm{M} n \mathrm{e}}
$$

where $N$ is the number of Bain's orientation variants, $(1 / N) \Phi_{n}$ is the volume fraction of the $n$-th variant of martensite, $\Phi^{\mathrm{gr}}=\frac{1}{N} \sum_{n=1}^{N} \Phi_{n}$ is the total volume fraction of martensite in a grain. Elastic strains of austenite $\varepsilon^{\mathrm{Ae}}$ and of the $n$-th variant of martensite $\varepsilon^{\mathrm{M} n}$ e are calculated by the Hook's law. Thermal strain $\varepsilon^{\text {gr T }}$ is calculated in a similar way with the use of isotropic expansion law for thermal strains of the two phases.

The phase strain of a martensite variant is the Bain's deformation $D^{n}$ realizing the transformation of the lattice and $(1 / N) \Phi_{n}$ is the weight of the $n$-th variant in the total phase strain. Thus,

$$
\varepsilon^{\mathrm{gr} P \mathrm{Ph}}=\frac{1}{N} \sum_{n=1}^{N} \Phi_{n} D^{n}
$$

Micro plastic strains are caused by the incompatibility of the phase strains. The main assumption for its calculation is that the phase strain of a Bain's variant activates a combination of slips producing a strain proportional to the deviator of the phase strain. Thus, for the total micro plastic strain of a grain one can write:

$$
\varepsilon^{\mathrm{gr} \mathrm{MP}}=\frac{1}{N} \sum_{n=1}^{N} \kappa \varepsilon_{n}^{\mathrm{p}} \operatorname{dev} D^{n}
$$

where internal variables $\varepsilon_{n}{ }^{\mathrm{p}}$ are measures of the microplastic strains, $\operatorname{dev} D^{n}$ is the deviator part of tensor $D^{n}, \kappa$ is a material constant.

\subsection{Transformation conditions}

To formulate the evolution equations for the variables $\Phi_{n}$ and $\varepsilon_{n}{ }^{\mathrm{p}}$ we consider generalized forces conjugated with these parameters. We start from the Gibbs' potential of the two-phase grain:

$$
G=\left(1-\Phi^{\mathrm{gr}}\right) G^{\mathrm{A}}+\frac{1}{N} \sum_{n=1}^{N} \Phi_{n} G^{\mathrm{M} n}+G^{\mathrm{mix}},
$$

where $G^{\mathrm{A}}$ and $G^{\mathrm{M} n}$ are the eigen potentials of austenite and $n$-th variant of martensite (potentials of the phases as if they were not interacting), $G^{\text {mix }}$ is the potential of mixing, which is the elastic inter-phase stress energy. In formula (6) the eigen potentials are:

$$
\begin{aligned}
G^{a}= & G_{0}^{a}-S_{0}^{a} \cdot\left(T-T_{0}\right)-\frac{c_{\sigma}^{a}\left(T-T_{0}\right)^{2}}{2 T_{0}}-\varepsilon_{i j}^{0 \mathrm{~T} a}(T) \sigma_{i j}- \\
& \frac{1}{2} D_{i j k l}^{a} \sigma_{i j} \sigma_{k l}, \quad a=\mathrm{A}, \mathrm{M} n
\end{aligned}
$$

superscript $a=A$ stands for austenite and $a=\mathrm{M} n$ - for the $n$-th variant of martensite; $T_{0}$ is the phase equilibrium temperature (i.e. such temperature, at which $G^{\mathrm{A}}=G^{\mathrm{M} n}$ ); $G_{0}{ }^{a}$ and $S_{0}{ }^{a}(a=\mathrm{A}, \mathrm{M} n)$ are the Gibbs' potentials and the entropies at stress $\sigma=0$ and temperature $T=T_{0} ; \varepsilon_{i j}{ }^{0 \mathrm{~T} a}(a=\mathrm{A}$, $\mathrm{M} n)$ are strains of the phases at $\sigma=0 ; c_{\sigma}{ }^{a}$ and $D^{a}{ }_{i j k l}(a=\mathrm{A}$, $\mathrm{M} n)$ are the specific heat capacities at constant stress and the elastic compliances. For $T_{0}$ we use an estimate proposed R.J. Salzbrenner, M. Cohen [6]: $T_{0}=(M \mathrm{~s}+A \mathrm{f}) / 2$ (hereinafter $M \mathrm{~s}, M \mathrm{f}, A \mathrm{~s}, A \mathrm{f}$ are the characteristic temperatures and $q_{0}$ is the latent heat of the transformation).

As for the elastic inter-phase stress energy $G^{m i x}$, it grows with the martensite variants fractions $\Phi_{n}$ and it is decreased by oriented defects $b_{n}$, produced by micro plastic deformations $\varepsilon_{n}{ }^{\mathrm{p}}$. In the works $[7,8]$ the authors considered martensite variants originated by an invariant plane deformation and characterized by the habit plane and the amount of shear. They suggested to calculate the energy of the variants interaction by a quadratic form. In the present work the same assumption is used for the interaction energy of the Bain's variants of martensite:

$$
G^{\text {mix }}=\frac{1}{2} \sum_{m, n=1}^{N} A_{m n}\left(\Phi_{m}-b_{m}\right)\left(\Phi_{n}-b_{n}\right),
$$

in which the diagonal components of matrix $A_{m n}$ describe the self-action and the off-diagonal elements - the interaction of the martensite variants. In TiNi alloy the basic self-accommodation of martensite is realized by grouping of the variants into correspondence variants pairs (CVP) [9-12]. We account for this fact by using the proper numeration of the variants and taking the matrix $A_{m n}$ in the form:

$$
A=\mu\left(\begin{array}{ccc}
A_{1} & & \\
& A_{1} & \\
& & A_{1}
\end{array}\right) ; \quad A_{1}=\left(\begin{array}{cccc}
1 & -\alpha & -\alpha & 0 \\
-\alpha & 1 & 0 & -\alpha \\
-\alpha & 0 & 1 & -\alpha \\
0 & -\alpha & -\alpha & 1
\end{array}\right)
$$

where the material constant $\alpha$ accounts for the interaction of the variants in a CVP, and $\mu=q_{0}\left((M \mathrm{f}-M \mathrm{~s}) / T_{0}\right) /(1-2 \alpha)$. The thermodynamic force causing growth of the $n$-th variant of martensite (i.e. increase of the variable $b_{n}$ ) is:

$$
\begin{aligned}
& F_{n}=-N \frac{\partial G}{\partial \Phi_{n}} \\
& \approx \frac{q_{0}}{T_{0}}\left(T-T_{0}\right)+\sigma_{i j} D_{i j}^{n}-\mu \sum_{m=1}^{N} A_{m n}\left(\Phi_{m}-b_{m}\right)
\end{aligned}
$$

There exists a dissipative force opposing the movement of the phase boundary and responsible for the thermal hysteresis of the transformation. Denoting this force by $F^{\mathrm{fr}}$, we formulate the transformation condition:

$$
F_{n}= \pm F^{\mathrm{fr}},
$$

where the plus sign is for the direct and minus - for the reverse transformation. The value of $F^{\mathrm{fr}}$ is derived from the transformation characteristics: $F^{\mathrm{fr}}=q_{0}\left(M \mathrm{~s}-T_{0}\right) / T_{0}$.

\subsection{Martensite reorientation}

A special approach is used to describe the reorientation (twinning) of martensite. This process is interpreted as a shift in the space of internal variables $\Phi_{1}, \ldots, \Phi_{N}$, such that the total amount of martensite $\Phi^{\mathrm{gr}}=\frac{1}{N} \sum_{n=1}^{N} \Phi_{n}$ is constant. We accept the following hypotheses.

1. Reorientation of martensite in a grain can occur only if this grain is purely martensitic $\left(\Phi^{\mathrm{gr}}=1\right)$. This hypothesis may seem too restrictive. Still, since reorientation requires a bigger stress than stress-induced transformation, it must become the dominating 
mechanism of deformation only when the direct transformation is completed.

2. Any variant of martensite can be transformed in any other variant.

3. Reorientation occurs along the direction in the space $\Phi_{1}, \ldots, \Phi_{N}$, which corresponds to the fastest decrease of the Gibbs' potential.

4. Reorientation starts when the thermodynamic force reaches some critical value.

To find the direction of the reorientation we use vector $F=\left\{-\frac{\partial G}{\partial \Phi_{1}}, \ldots,-\frac{\partial G}{\partial \Phi_{n}}\right\}$ and take its projection $L$ onto plane $\Phi_{1}+\ldots+\Phi_{N}=$ const. Then if for some $n$ it holds that $\Phi_{n}=0$ and $L_{n}<0$ we substitute $L$ for its projection $L^{\prime}$ onto intersection of planes $\Phi_{n}=0$ and $\Phi_{1}+\ldots+\Phi_{N}=$ const repeating this procedure for other components of $L_{n}$ if necessary. Finally we obtain the unit direction $l$, which does not lead to a violation of conditions $\Phi_{n}>0, n=1, \ldots, N$. For this direction we postulate the condition of reorientation:

$$
F^{\mathrm{tw}}(l)=F^{\mathrm{fr} \mathrm{tw}}
$$

where

$$
F^{\mathrm{tw}}(l)=-\frac{\partial G}{\partial l}=-\sum_{n=1}^{N} l_{n} \frac{\partial G}{\partial \Phi_{n}}=N \sum_{n=1}^{N} l_{n} F_{n}
$$

In (11) $F^{\mathrm{fr} \text { tw }}$ is a constant, characterizing the critical force for reorientation. From hypotheses 2 and 3 it follows that the increments $d \Phi_{n}$ are proportional to $l_{n}$ :

$$
d \Phi_{n}=l_{n} d \varphi
$$

where $d \varphi$ is the proportionality factor to be found from condition (11).

\subsection{Micro plastic flow conditions}

Conditions (10) or (11) are insufficient for determination of the increments of all internal variables. To find the variation law of variables $b_{n}$ we formulate the microplastic flow conditions:

$$
\left|F_{n}{ }^{\mathrm{p}}-F_{n}{ }^{\rho}\right|=F_{n}{ }^{\mathrm{y}}, \quad d\left|F_{n}{ }^{\mathrm{p}}\right|>0,
$$

where $F_{n}{ }^{\mathrm{p}}$ is the generalized force conjugated with the parameters $b_{n}$ :

$$
F_{n}^{\mathrm{p}}=-N \frac{\partial G}{\partial \Phi_{n}^{p}}=\mu \sum_{m=1}^{N} A_{m n}\left(\Phi_{m}-b_{n}\right),
$$

$F_{n}{ }^{\mathrm{y}}$ and $F_{n}{ }^{\rho}$ are the forces describing the isotropic and kinematic hardening. Note that the micro-plastic flow condition (14) is analogous to the classic plastic flow condition for $1 \mathrm{D}$ case, forces $F_{n}{ }^{\mathrm{p}}$ playing the role of the stress and $F_{n}{ }^{\mathrm{y}}, F_{n}{ }^{\rho}$ - the roles of the flow stress and the back stress respectively. Deformation defects generated by the micro plastic flow we divide in two groups: oriented defects $b_{n}$ and scattered defects $f_{n}$, suggesting the evolution equations for them in the form:

$$
\dot{b}_{n}=\dot{\varepsilon}_{n}^{\mathrm{p}}-\frac{1}{\beta^{*}}\left|b_{n}\right| \dot{\varepsilon}_{n}^{\mathrm{p}} H\left(b_{n} \dot{\varepsilon}_{n}^{\mathrm{p}}\right), \quad \dot{f}_{n}=\left|\dot{\varepsilon}_{n}^{\mathrm{p}}\right|
$$

where $\beta^{*}$ is a material constant. First of the equations (16) reflect the fact that oriented defects grow in a proportional way with the micro plastic deformation, this growth being limited by escape to the outer surface. The second equation (16) means that scattered defects are proportional to the summary plastic deformation (Odquist parameter). We assume that the scattered defects give rise to the isotropic hardening of the material and the oriented ones - to the kinematic hardening: we relate the defect densities $f_{n}$ and $b_{n}$ to $F_{n}{ }^{\mathrm{y}}$ and $F_{n}{ }^{\rho}$ by the so called closing equations, which we choose in the simplest linear form:

$$
F_{n}^{\mathrm{y}}=a_{\mathrm{y}} f_{n}, \quad F_{n}^{\rho}=a_{\rho} b_{n},
$$

where $a_{\mathrm{y}}$ and $a_{\mathrm{\rho}}$ are material constants. From conditions (10) and (14) in the case of the transformation or (11) and (14) in the case of martensite reorientation using formulae (9), (12), (13), (15) - (17) evolution equations relating the increments of the internal variables $\Phi_{n}, b_{n}, f_{n}$ and $\varepsilon_{n}^{\mathrm{p}}$ to the increments of stress and temperature are derived. Formulae (1) - (5) allow calculating the reversible and irreversible macroscopic strain.

\section{Simulation results}

The values of the material constants specifying the elastic, thermal and phase deformation of SMA were

\begin{tabular}{|c|c|}
\hline Material constant & Value \\
\hline $\begin{array}{l}\text { Characteristic } \\
\text { temperatures } M \mathrm{f}, \quad M \mathrm{~s}, \\
A \mathrm{~s}, A \mathrm{f}\end{array}$ & $317,326,397,406 \mathrm{~K}$ \\
\hline Latent heat $q_{0}$ & $-160 \mathrm{MJ} / \mathrm{m}^{3}$ \\
\hline $\begin{array}{l}\text { Number of martensite } \\
\text { variants } N\end{array}$ & 12 \\
\hline $\begin{array}{l}\text { Lattice deformation } \\
\text { matrix } D[14]\end{array}$ & $\left(\begin{array}{ccc}0.0188 & 0.0562 & 0.0488 \\
0.0562 & 0.0188 & 0.0488 \\
0.0488 & 0.0488 & -0.0369\end{array}\right)$ \\
\hline $\begin{array}{l}\text { Elastic modulus of } \\
\text { austenite } E_{\mathrm{A}}\end{array}$ & $80 \mathrm{GPa}$ \\
\hline $\begin{array}{l}\text { Elastic modulus of } \\
\text { martensite } E_{\mathrm{M}}\end{array}$ & $25 \mathrm{GPa}$ \\
\hline $\begin{array}{lll}\text { Poisson's } & \text { ratio } & \text { of } \\
\text { austenite } v_{\mathrm{A}} & & \end{array}$ & 0.33 \\
\hline $\begin{array}{l}\text { Poisson's ratio of } \\
\text { martensite } v_{M}\end{array}$ & 0.45 \\
\hline Thermal-expansion & $11 \cdot 10^{-6} \mathrm{~K}^{-1}$ \\
\hline
\end{tabular}
chosen to reproduce the mechanical behavior of the equiatomic TiNi SMA. For a specific TiNi composition experimentally studied in [13] they were determined in calorimetric and mechanical tests. The values of all constants are collected in Table 1.

Table 1. Values of the material constants. 


\begin{tabular}{|c|c|}
\hline coefficient of austenite & \\
\hline $\begin{array}{l}\text { Thermal-expansion } \\
\text { coefficient of martensite }\end{array}$ & $6.6 \cdot 10^{-6} \mathrm{~K}^{-1}$ \\
\hline $\begin{array}{ll}\text { Variants } & \text { interaction } \\
\text { constant } \alpha & \end{array}$ & 0.2 \\
\hline $\begin{array}{l}\text { Critical reorientation } \\
\text { force } F^{\text {fr tw }}\end{array}$ & $20 \mathrm{MJ} / \mathrm{m}^{3}$ \\
\hline $\begin{array}{l}\text { Micro plastic } \\
\text { scaling factor } \kappa\end{array}$ & 1.9 \\
\hline $\begin{array}{ll}\text { Isotropic hardening } \\
\text { factor } a_{\mathrm{y}}\end{array}$ & $0.2 \mathrm{MPa}$ \\
\hline $\begin{array}{l}\text { Kinematic hardening } \\
\text { factor } a_{\rho}\end{array}$ & $5 \mathrm{MPa}$ \\
\hline $\begin{array}{l}\text { Oriented defects } \\
\text { saturation factor } \beta^{*}\end{array}$ & 2.2 \\
\hline
\end{tabular}

b)

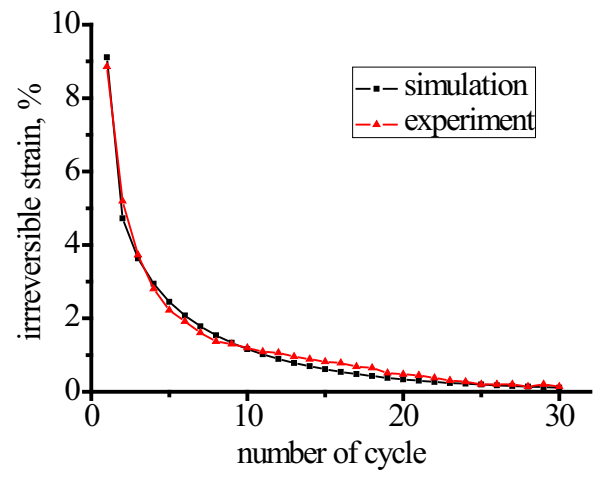

Figure 2. Simulated and experimental [13] dependences of the irreversible strain increment in one thermocycle under stress $50 \mathrm{MPa}$ (a) and $200 \mathrm{MPa}$ (b) on the cycle number.

The irreversible strain in one cycle decreases with the number of cycles tending to some small value. The results of simulation well agree with the experiment for stress $200 \mathrm{MPa}$. Not so good agreement for stress $50 \mathrm{MPa}$ shows that some relaxation process reducing the deformation hardening are not taken into account.

Active SMA parts in actuators usually experience thermomechanical cycles in which the stress on heating $\sigma_{\mathrm{h}}$ is set commonly at bigger values than the stress on cooling $\sigma_{\mathrm{c}}$. Naturally, the irreversible strain grows with the applied stress. An example of the simulation of the dependence of irreversible strain on the stress $\sigma_{\mathrm{h}}$ is shown in Figure 3.
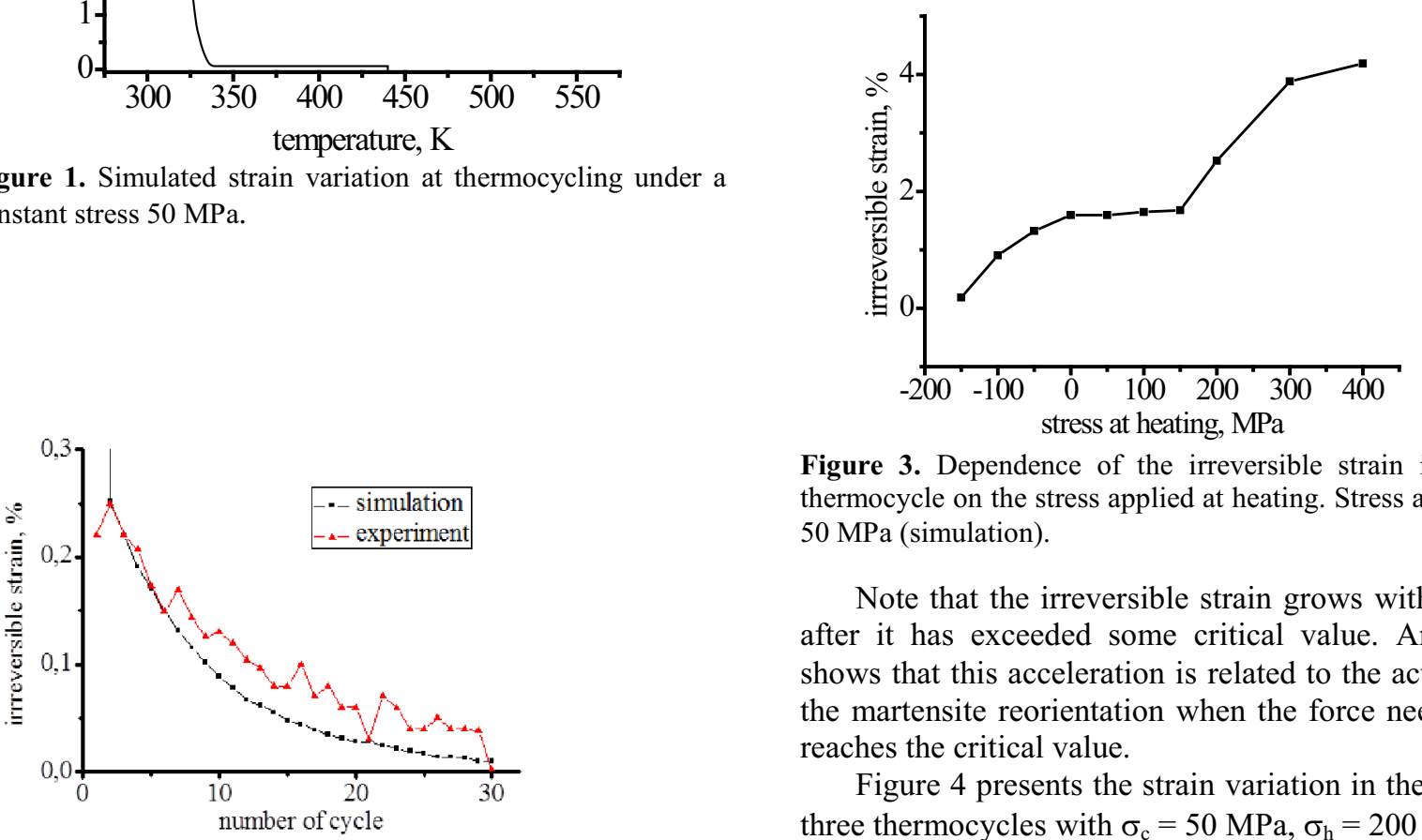

Figure 3. Dependence of the irreversible strain in the first thermocycle on the stress applied at heating. Stress at cooling is $50 \mathrm{MPa}$ (simulation).

Note that the irreversible strain grows with $\sigma_{\mathrm{h}}$ faster after it has exceeded some critical value. An analysis shows that this acceleration is related to the activation of the martensite reorientation when the force needed for it reaches the critical value.

Figure 4 presents the strain variation in the course of three thermocycles with $\sigma_{\mathrm{c}}=50 \mathrm{MPa}, \sigma_{\mathrm{h}}=200 \mathrm{MPa}$. 


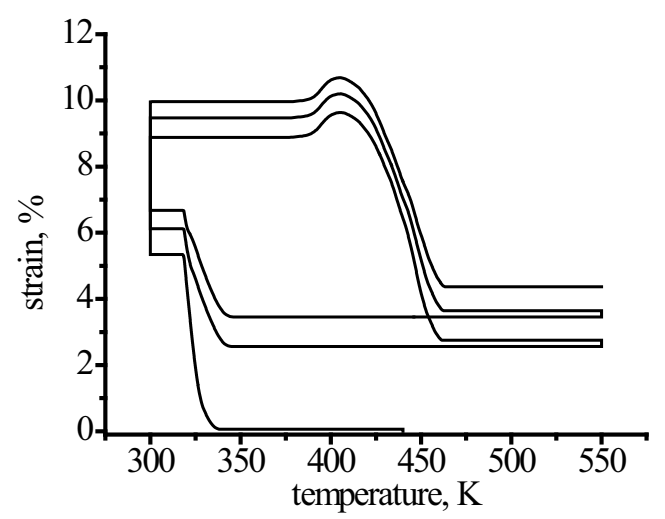

Figure 4. Strain variation under thermocycling at stress on cooling $\sigma_{\mathrm{c}}=50 \mathrm{MPa}$ and stress on heating $\sigma_{\mathrm{h}}=200 \mathrm{MPa}$ (simulation).

One can see a typical "hump" on the strain dependence on temperature while heating. It is related to the reorientation of martensite occurring along the sequence "martensite - virtual austenite - martensite of other orientation" prior to the start of the reverse transformation.

Figure 5 shows the growth of the total irreversible strain on thermocycing. The irreversible strain after 30 cycles under $\sigma_{\mathrm{c}}=50 \mathrm{MPa}, \sigma_{\mathrm{h}}=200 \mathrm{MPa}$ is only two times larger than after 30 cycles under $\sigma_{\mathrm{c}}=\sigma_{\mathrm{h}}=50 \mathrm{MPa}$. At the same time cycling under $\sigma_{\mathrm{c}}=\sigma_{\mathrm{h}}=200 \mathrm{MPa}$ results in a much bigger growth of the irreversible strain.

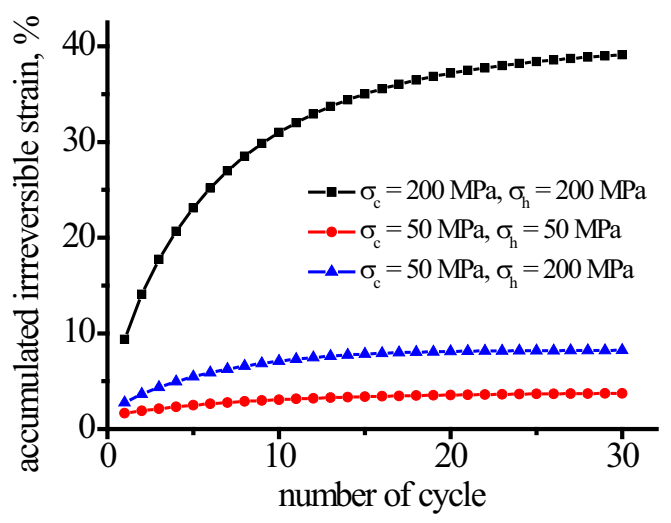

Figure 5. Dependence of the total accumulated irreversible strain on the number of thermocycles carried out under different stresses $\sigma_{\mathrm{c}}$ applied on cooling and $\sigma_{\mathrm{h}}$ applied on heating (simulation).

\section{Summary}

Incorporation of the deformation defects conception into a microstructural model opens the possibility of simulating the irreversible strain acquired by an SMA specimen at thermomechanical cyclic loading under different stresses applied on the stages of cooling and heating. An account of the deformation defects production and of their effect on the isotropic and kinematic hardening allows describing the decrease of the irreversible strain in one cycle with the cycle number. This data is important for designing of SMA actuators.
Simulation predicts that the total irreversible strain growth must be influenced stronger by the stress applied on cooling than on heating.

\section{Acknowledgements}

This research was supported by the grant of Russian Foundation of Basic Research 15-01-07657.

One of the authors (A.E. Volkov) acknowledges SaintPetersburg State University (Russia) support of presenting this work at the conference ESOMAT-2015 (project 6.41.647.2015).

\section{References}

1. Q.-P. Sun, C. Lexcellent, J. de Physique IV. 6 (1996), C1-367-375.

2. M.E. Evard 121, A.E. Volkov, J. Eng. Mater. and Technol. (1999), 102-104.

3. A.E. Volkov, F. Casciati, in: F.Auricchio, L.Faravelli, G.Magonette and V.Torra (Eds.) Shape Memory Alloys. Advances in Modelling and Applications, Barcelona, 88-104 (2001).

4. F.S. Belyaev, M.E.Evard, A.E. Volkov, N.A. Volkova, Materials Today Proceedings. (to be published)

5. A.E. Volkov, M.E. Evard, F.S. Belyaev, Materials Science Foundations, 81-82 (2015), 20-37.

6. R.J. Salzbrenner, M. Cohen, Acta Met., 27, N 5 (1979), 739-748.

7. N. Siredey, E. Patoor, M. Berveiller, A. Eberhardt, Int. J. of Solids and Structures 36 (1999), 4289-4315.

8. Y. Chemisky, A. Duval, E. Patoor, T. Ben Zineb, Mechanics of Materials 43 (2011) 361-376.

9. K. Madangopal, J. Singh, Benerjee, Scr. Met., 25, 2153-2158 (1991)

10. K. Madangopal, J. Singh, Acta Mater., 48 (2000), 1325-1344.

11. M. Nishida, T. Nishiura, H. Kawano, T. Imamura, Phil. Mag., 92 (2012), 2215-2233.

12. M. Nishida, E. Okunishi, T. Nishiura, H. Kawano, T. Imamura, S. Ii, T. Hara, Phil. Mag., 92 (2012), 2234-2246.K.M. Knowles, D.A. Smith, Acta Met., 20, 101-110 (1981)

13. S. Belyaev, N. Resnina, A. Sibirev, J. Mater. Eng. Perform., 23 2339-2342 (2014).

14. T. Imamura, T. Nishiura, H. Kawano, H. Hosoda, M. Nishida, Phil. Mag., 92 (2012), 2247-2263. 